\title{
The ISO/ISPO classification of congenital limb deficiency
}

\author{
H. J. B. DAY \\ Formerly of Disablement Services Centre, Withington Hospital, Manchester, UK
}

\begin{abstract}
This classification originally produced by the ISPO "Kay" Committee in 1973, has now with minor modifications, become an International Standard (ISO 8548-1: 1989). It is limited to those deficiencies which are failures of formation and describes them on anatomical and radiological bases only. All are divided into transverse and longitudinal, and use simple terms and descriptors.
\end{abstract}

\section{Introduction}

A logical system of classification and nomenclature is needed to facilitate scientific communication about congenital limb deficiency. The lack of a suitable system has allowed the use of the term "congenital amputation" - implying that a limb segment has been lost before birth - to be used for cases which are patently failures of formation. Furthermore any classification should use simple words capable of translation into all languages. The use of terms derived from Greek or Latin roots may sound impressively scientific, but they are both inaccurate and ambiguous, and are often misused, none more frequently than "phocomelia" which is used to describe every level and type of deficiency.

The history of classifications devised since that of Frantz and O'Rahilly (1961), including those of Burtch (1966), Henkel and Willert (1969) and the work of the ISPO "Kay" committee has been described previously by Kay (1974), Swanson (1976) and Day (1988).

The ISPO system provided the framework which enabled the Working Group of ISO Technical Committee 168 (Prosthetics and

All correspondence to be addressed to Tithe House, Over Peover, Knutsford, Cheshire WA16 8UG, UK.
Orthotics) to set out a proposal for an International Standard. This has been accepted by the participating nations and has been published as an International Standard, ISO 8548-1: 1989 "Method of describing limb deficiencies at birth".

\section{ISO 8548-1: 1989}

The Standard has three constraints:-

1. The classification is restricted to skeletal deficiencies and therefore the majority of such cases are due to a failure of formation of parts.

2. The deficiencies are described on anatomical and radiological bases only. No attempt is made to classify in terms of embryology, aetiology or epidemiology.

3. Classically derived terms such as hemimelia, peromelia, etc., are avoided because of their lack of precision and the difficulty of translation into languages which are not related to Greek.

Deficiencies are described as Transverse and Longitudinal.

The former resemble an amputation residual limb, in which the limb has developed normally to a particular level beyond which no skeletal elements are present. All other cases are classed as longitudinal in which there is reduction or absence of an element or elements within the long axis of the limb.

\section{Method of description}

\section{Transverse}

The limb has developed normally to a particular level beyond which no skeletal elements exist, although there may be digital buds. Such deficiencies are described by naming the segment at which the limb terminates and then describing the level within the segment beyond which no skeletal elements exist (Table 1). 
It is possible to use another descriptor in the phalangeal case to indicate a precise level of loss within the fingers.

Table 1. Designation of levels of transverse deficiencies of upper and lower limbs.

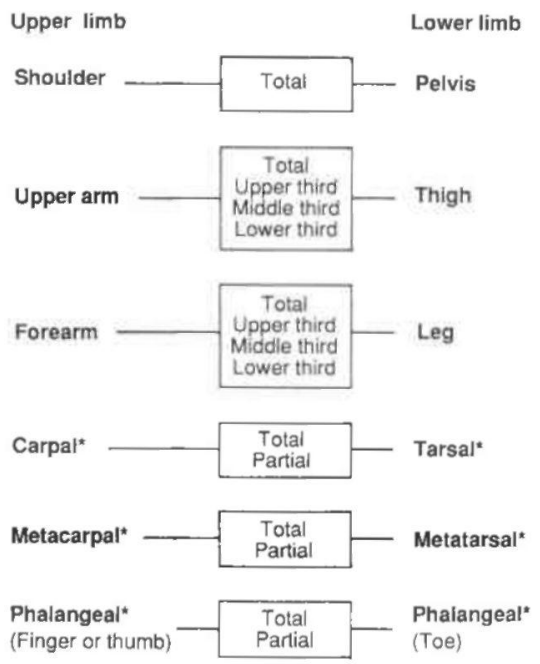

Notes:

(1) The skeletal elements marked * are used as adjectives in describing transverse deficiencies, e.g. transverse carpal total deficiency.

(2) Total absence of the shoulder or hemipelvis (and all distal elements) is a transverse deficiency. If only a portion of the shoulder or hemipelvis is absent, the deficiency is of the longitudinal type.

Table 2. Description of longitudinal deficiencies of the upper limb.

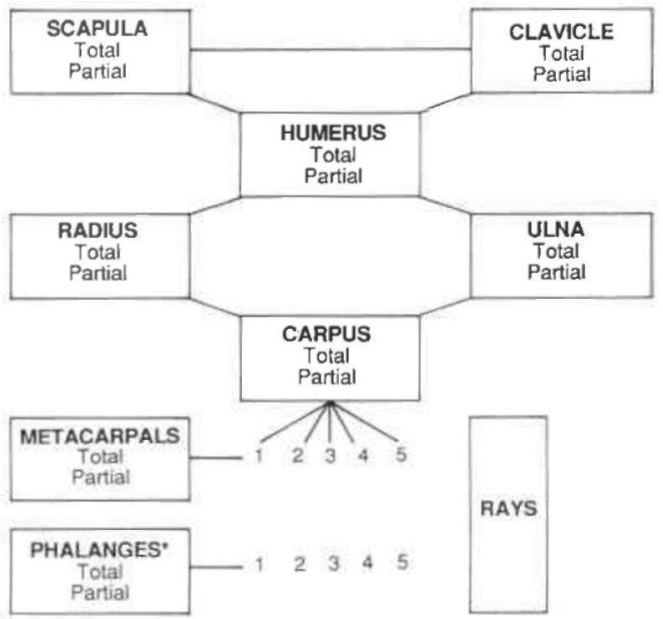

* The digits of the hand are sometimes referred to by name:- 1 , thumb; 2 index; 3 , middle; 4 , ring; and 5 , little (or small). For the purpose of this classification such naming is deprecated because it is not equally applicable to the foot.

\section{Longitudinal}

There is a reduction or absence of an element or elements within the long axis of the limb and in this case there may be normal skeletal elements distal to the affected bone or bones. To describe such a deficiency refer to Tables 2 and 3 and follow the procedure below:

1. Name the bones affected, in a proximodistal sequence, using the name as a noun. Any bone not named is present and of normal form.

2. State whether each affected bone is totally or partially absent.

3. In the case of partial deficiencies the approximate fraction and the position of the absent part may be stated.

4. The number of the digit should be stated in relation to a metacarpal, a metatarsal and the phalanges, the numbering starting from the preaxial, radial or tibial side.

5. The term "Ray" may be used to refer to a metacarpal or metatarsal and its corresponding phalanges.

Examples of transverse and longitudinal deficiencies are shown in Figures 1 and 2, but it must be understood that the stylised representation of the limb which is used in these figures is neither part of the original ISPO "Kay" committee work nor of the new

Table 3. Description of longitudinal deficiencies of the lower limb.

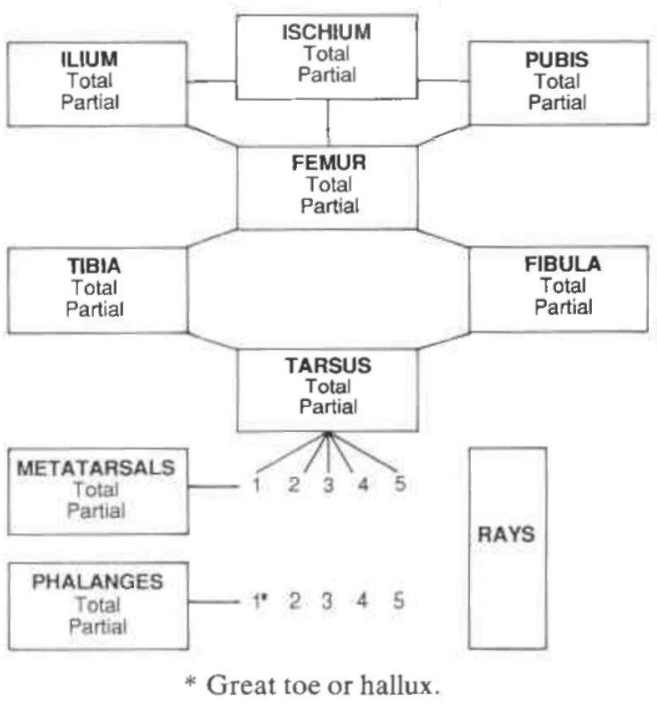




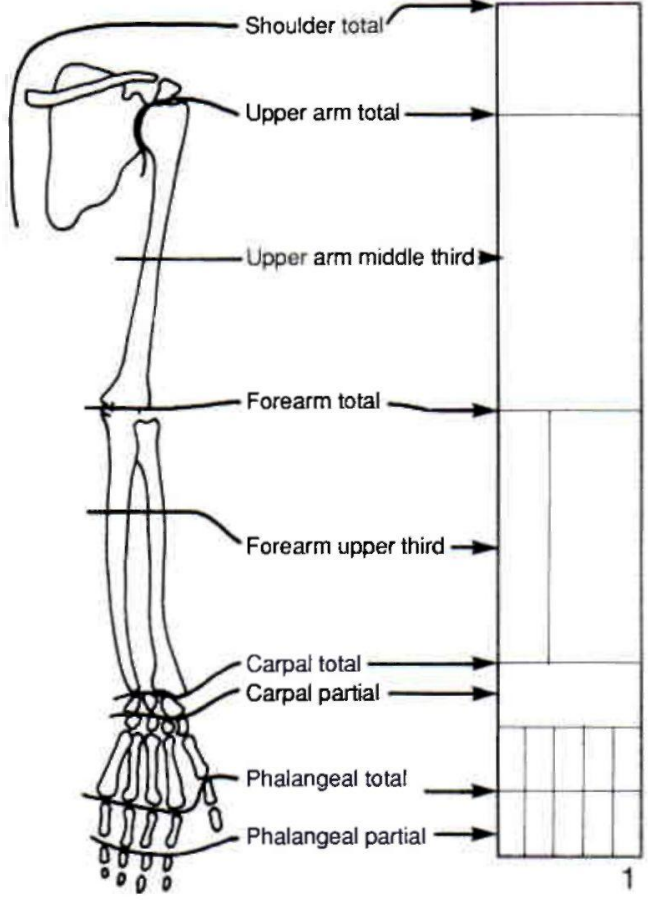

Fig. 1. Examples of transverse deficiencies at various levels, shown on the skeleton and as the author's stylised representation.

International Standard, but the author has found it to be the most useful way of illustrating deficiencies in clinical notes and it can be used to indicate some treatment as well as the deficiency.

\section{Acknowledgement}

Parts of ISO 8548-1: 1989 are reproduced here with the permission of the International Organisation for Standardisation (ISO). Copies of this standard are available from the ISO Central Secretariat, Case Postale 56, CH-1211 Geneva 20, Switzerland or from any ISO member body.

\section{REFERENCES}

Burtch, R. L. (1966). Nomenclature for congenital skeletal limb dificiencies, a revision of the Frantz and O'Rahilly classification. Artificial Limbs, 10(1), 24-35.

Frantz, C. H., O'Rahilly, R. (1961). Congenital skeletal limb deficiencies. J. Bone Joint Surg., 43A, 1202-1204.
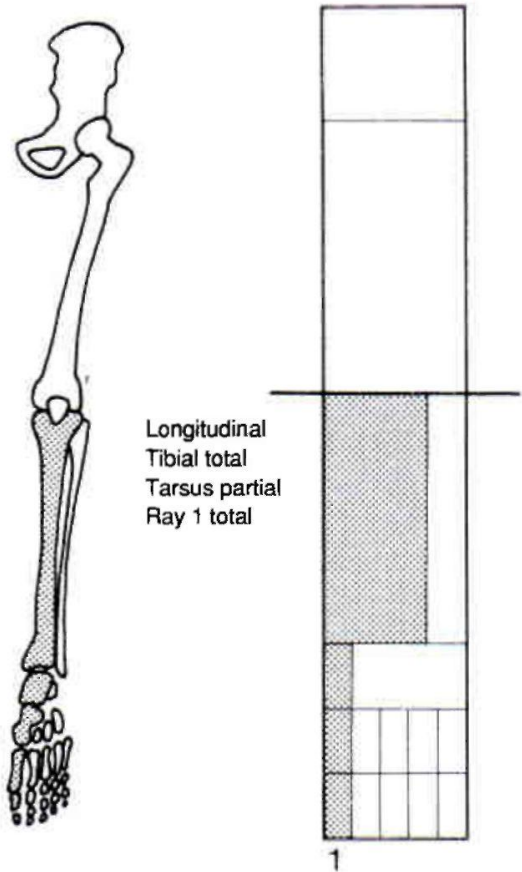

Fig. 2. Example of a longitudinal deficiency shown on the skeleton and as the author's stylised representation, showing not only the original deficiency but also the treatment by knee disarticulation.

DAY, H. J. B. (1988). Nomenclature and classification in congenital limb deficiency. In: Amputation surgery and lower limb prosthetics. Edited by Murdoch. G. Blackwell. - Edinburgh, Blackwell Scientific. p271-278.

Henkel, H. L., Willert H. G. (1969). Dysmelia, a classification and a pattern of malformation of congenital limb deficiencies. J. Bone Joint Surg., 51B, 399-414.

ISO 8548-1: 1989. Prosthetics and Orthotics - Limb deficiencies - Part 1: Method of describing limb deficiencies present at birth.

KAY, H. W. (1974). A proposed international terminology for the classification of congenital limb deficiencies. ICIB, 13(7), 1-16.

KAY, H. W. (1975). The proposed international terminology for the classification of congenital limb deficiencies. The recommendations of a working group of ISPO. Dev. Med. Child Neurol. Suppl., 34, 1-12.

Swanson A. B. (1964). A classification for congenital malformations of the hand. N. J. Bull., Acad. Med., 10, 166-169.

Swanson A. B. (1976). A classification for congenital limb malformations. J. Hand Surg., 1, 8-22. 